\title{
Flow Stress of beryllium: Attempt for a Bayesian Crossed-data Analysis from Hopkinson Bars to Rayleigh-Taylor Instabilities
}

\author{
Gabriel Seisson ${ }^{1, *}$, Vincent Dubois ${ }^{1}$, Cyril Bolis $^{1}$, and Christophe Denoual ${ }^{1}$ \\ ${ }^{1}$ CEA/DIF, Bruyères-le-Châtel, F-91297 Arpajon, France
}

\begin{abstract}
In this paper, we demonstrate by a Bayesian approach the incapacity of the Preston-Tonks-Wallace (PTW) strength model to represent, with the same set of parameters, the flow stress of beryllium in both moderate and highly dynamic experiments, and suggest hypotheses explaining that limitation. Usual plasticity models such as Johnson-Cook (JC) and PTW are mostly adjusted onto quasi-static and dynamic uni-axial compression data acquired thanks to compression machines and split Hopkinson pressure bars. Nonetheless, they may be used beyond the range of mechanical loading in which they have been fitted. This is the case of the simulations of solid Rayleigh-Taylor instabilities (RTI) driven by high explosives. A recent work of Henry de Frahan et al. noticed the inability of various plasticity models to stand for the growth of beryllium RTI. Amongst them, the PTW model has been particularly examined through four different sets of parameters, each of them largely underestimates the growth of the experimental instability. Thus, this work is an attempt, regarding the plastic flow modeling of beryllium, to conciliate uni-axial compression tests (CT) and RTI by means of a crossed Bayesian analysis.
\end{abstract}

\section{Introduction}

In 1974, Barnes et al. [1] demonstrated the relevance of Rayleigh-Taylor instabilities (RTI) driven by high explosive (HE) to examine the dynamic plastic behavior of metals (aluminum or steel). It consisted in using the detonation products of high explosives (the light medium) to accelerate without shock metallic plates (the heavy medium) with sinusoidal perturbations machined on the surface facing the HE. The growth of the original perturbation, which is directly linked to the material strength, was observed along the experiment using transverse XRAY flash. A few decades later, Park et al. [2,3] conducted similar experiments with lasers. In this case, the plasma generated by the interaction of a $\mathrm{CH}$ reservoir and the laser beam acted as the light medium accelerating the heavy one (vanadium or tantalum). The perturbation growth was measured by face-on XRAY radiographies.

Recently, Henry de Frahan et al. [4] investigated the plastic behavior of beryllium S200F under dynamic loading thanks to HE-driven Rayleigh-Taylor instabilities (see figure 1). A two-stage planar HE drive accelerated an iron flyer impacting a charge of HE placed at a few millimeters from the 4-mm-wavelength $(\lambda)$ and 0.48 -mm-amplitude $\left(A_{0}\right)$ sinusoidal front face of a 2-mm-thick $(h)$ target made of beryllium S200F.

The experiment was repeated four times and for each of them, a transverse radiography was made at a different time giving the perturbation growth $\left(A / A_{0}\right)$ according to the target's back face displacement $(S)$. The RING [5], SCG [6], SL [7] and MTS [8] plasticity mod-

\footnotetext{
*e-mail: gabriel.seisson@ cea.fr
}

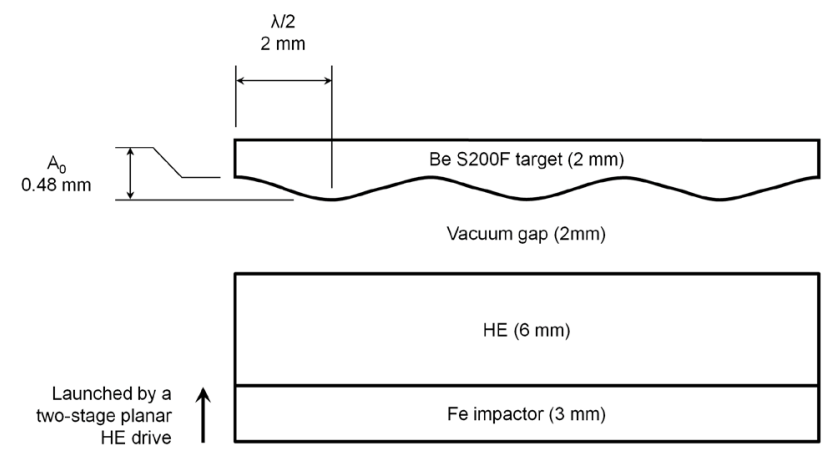

Fig. 1. Experimental set-up of the Henry de Frahan's HE-driven RTI on beryllium S200F [4]. $\lambda$ : perturbations' wavelength. $A_{0}$ : perturbations' initial amplitude.

els were tested. Figure 2 evidences that none of them was able to reproduce the experimental growth factor (GF). Furthermore, four different sets of parameters of the Preston-Tonks-Wallace model (PTW) [9] adjusted on uni-axial compression experiments from compression machines (CM) and split Hopkinson pressure bars (SHPB) were tested. PTW takes into account the effects of strain hardening, strain rate and temperature and possible athermal plateau, thermally activated phenomena and phonon drag regime. It is well suited for BCC materials such as iron (or steel), tantalum and molybdenum but can also be used for FCC materials such as aluminum and copper. It has also been used by its own authors for other crystal structures including orthorhombic (uranium) and hexagonal (beryllium) [9]. However, in the Henry de Frahan's study, none of the four PTW sets succeeded to match the 
experiment, giving results as disappointing as the other models. It suggests that either the experimental uncertainties must be taken into account during the adjustment process on CM and SHPB experiments in order to be able to match the RTI results too, or the PTW model cannot account for particular strain mechanisms of beryllium through large loading ranges.

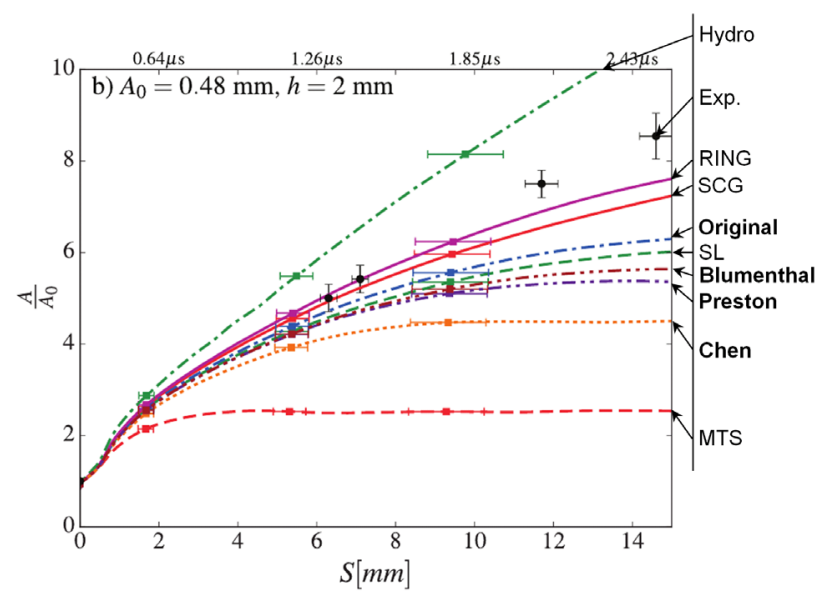

Fig. 2. Adapted from [4]. Growth factor $A / A_{0}$ vs. back face displacement $S$. All the tested strength models underestimate the experimental results. Bold text: the four PTW sets.

The present paper is an attempt to obtain through a Bayesian approach a PTW set of parameters for beryllium S200F that can be used for both uni-axial compression tests and Rayleigh-Taylor instability experiments. We conduct two separated Bayesian calibrations, the first on the compression tests (CT) from the Blumenthal's database [10] considering upper-bounding experimental errors, and the second on the RTI thanks to a surrogate model. We evidence that the parameters' posterior 95\%confidence intervals (CI) do not intersect, a fact that underlines the inability of PTW to account simultaneously for CT and RTI. Finally, we discuss some possible origins of this mismatch.

\section{$2 \mathrm{CM}$ and SHPB experiments}

Concerning beryllium S200F, there are two well-known database of uni-axial compression tests respectively published by Blumenthal [10] and Montoya [11]. The first has been chosen for this study since it presents the largest ranges in terms of stress $(\sigma)$, strain rate $(\dot{\epsilon})$ and temperature $(T)$, especially with a dynamic test at $77 \mathrm{~K}$.

\subsection{Blumenthal's database}

Figure 3 compares this database to the PTW set of parameters proposed in the same study and so called PTW Blumenthal in reference [4]. Without any evaluation of the experimental errors by the authors, we choose a standard deviation equal to $6 \%$ of the flow stress for SHPB experiments (adiabatic) and 12\% for the CM tests (isotherm) which are reasonable upper-bounding values. It appears clearly that the set PTW Blumenthal could be improved.

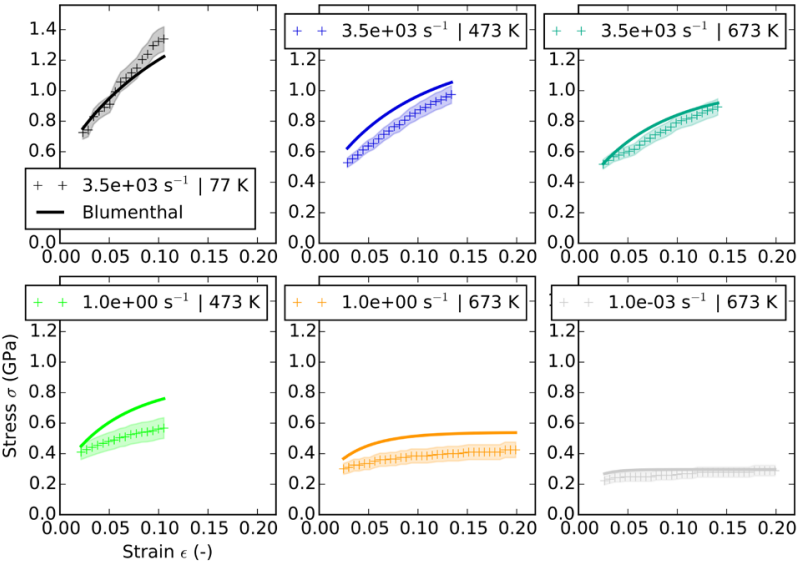

Fig. 3. Comparison experiment-simulation for the Blumenthal's database [10]. (+): experiments. Shadows: experimental standard deviation (see text for details). (-): simulations with PTW Blumenthal.

\subsection{Priors and sensitivity analysis}

From various PTW sets for beryllium S200F collected in unpublished data and references [4, 12], priors of seven parameters are set as Uniform laws in the intervals summarized in table 1 . Note that the parameters of the shear modulus are not considered here because in the PTW model they already affect the values of $s_{0}, s_{\infty}, y_{0}$ and $y_{\infty}$ through their respective normalization. Phonon drag regime is also neglected since it is encountered neither in the compression tests nor during the RTI experiments since the transition is supposed to be at $\dot{\epsilon} \approx 10^{7} \mathrm{~s}^{-1}$.

Table 1. Bounds of the Uniform probability laws used as priors for the seven PTW parameters considered in this study.

\begin{tabular}{ccccccc}
\hline$\theta$ & $\psi$ & $s_{0}$ & $s_{\infty}$ & $\kappa$ & $y_{0}$ & $y_{\infty}$ \\
\hline 0.025 & 0 & 0.003 & 0.0005 & 0.1 & 0.0005 & 0.0003 \\
0.045 & 3 & 0.023 & 0.0014 & 0.5 & 0.0022 & 0.0010 \\
\hline
\end{tabular}

A weight is attributed to each Blumenthal's compression test. High stain rates being closer to the thermomechanical conditions of the RTI, tests at $3500 \mathrm{~s}^{-1}$ have a weight of two. Moreover, according to PTW there is a time-temperature equivalence making the experiment at $77 \mathrm{~K}$ representative of higher strain rates at moderate temperatures. It thus receives a weight of four whereas the tests at lower strain rates get a weight of one.

Before any Bayesian calibration, the most influent parameters are usually found by means of a sensitivity analysis such as Morris' or Sobol's. It allows us to reduce the number of calibrated parameters and thus to save computing time. The results of a Morris sensitivity analysis is plotted in figure 4 . It reveals that two parameters ( $\psi$ and $\theta$ ) have a very small influence on the compression tests. Five other parameters $\left(s_{0}, \kappa, y_{0}, y_{\infty}\right.$ and $\left.s_{\infty}\right)$ have moderate or strong influence with correlation to each other. Therefore, the latter are chosen for the Bayesian calibration and $\psi$ and $\theta$ are set to their best values given by a maximum likelihood process whose results are given in the left hand side table of figure $8(\mathrm{~b})$. 


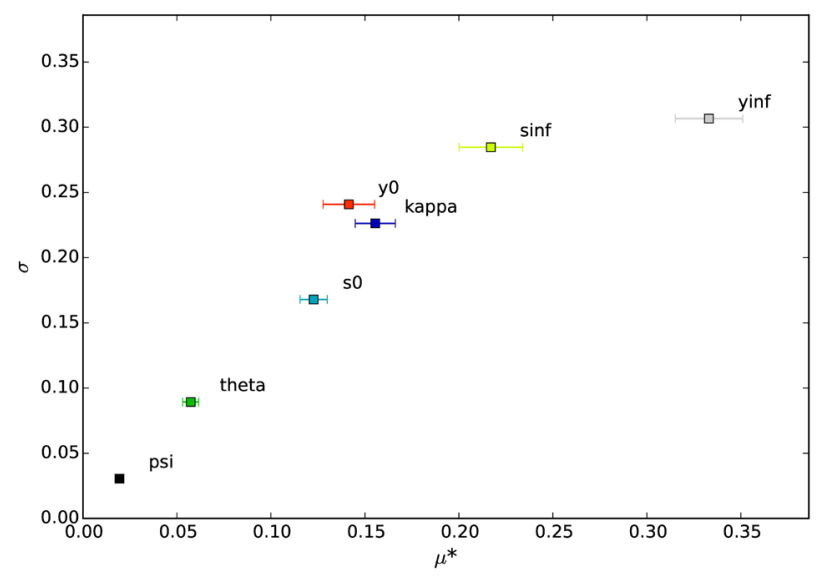

Fig. 4. Results of the Morris' sensitivity analysis for the Blumenthal's database with the intervals from table 1. $\mu^{*}$ accounts for parameter's average sensitivity. $\sigma$ accounts for parameter's correlation to the others and non-linear sensitivity.

\subsection{Bayesian calibration}

A Bayesian calibration is performed (less than 4 hours, 1 CPU) using a 2-million-length Markov's chain and an adaptive Metropolis-Hastings' algorithm on the weighted (see sec. 2.1) Blumenthal's tests. For each of them, we consider a Normal law defined by the maximum likelihood of the experimental stress and its standard deviation given in section 2.1. The priors of the five parameters $s_{0}, s_{\infty}, \kappa$, $y_{0}$ and $y_{\infty}$ are those from table 1 . The left hand side table of figure 8 (b) gives their posterior 95\%-CI. In addition, figure 8(a) (same side) displays the posterior 95\%-CI of the strain-stress curves which appear to be in a very good agreement with the experiments being largely bounded by the experimental intervals.

\section{RTI experiments}

Following the numerical details of Henry de Frahan et al. [4], we simulated half a wavelength of the RTI experiment in a 2D-planar geometry with the Eulerian mode of the Hésione hydrocode. We used a $10-\mu \mathrm{m}$ mesh size and a failure stress of $1.1 \mathrm{GPa}$ [11] whereas the authors used an Arbitrary Lagrangian-Eulerian code (named Ares) with $8-\mu \mathrm{m}$ mesh size without any damage model. Only at these conditions, we were able to obtain the same growth factors as Henry de Frahan for each of the four PTW sets. Figure 5 plots results for the so-called set PTW Original. To match the experiment, it appears that the flow stress must be divided by about two (0.45: best fit ; 0.40 and 0.55 : lower and upper bounds) in every cell of the simulation at every time step. Although this method lead to a good agreement with the experiment, it only shows the path to follow and the magnitude of changes to adapt the set of parameters. Nonetheless, such hydrodynamic simulations take around five hours on $128 \mathrm{CPU}$ each thus, before any direct optimization or Bayesian calibration, this cost has to be strongly reduced. To do so, we choose to define a surrogate model, which could be evaluated at a very reduced cost, as defined in the next section.

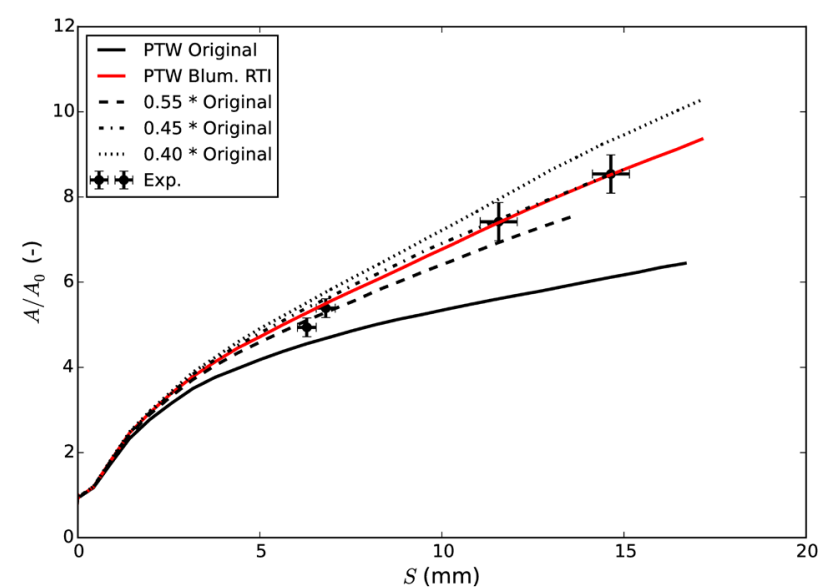

Fig. 5. Comparison between experiments from reference [4] and Hésione simulations with PTW Original. Multiplying by 0.45 the flow stress calculated by PTW allows to correctly match the experiment. Coefficients of 0.40 and 0.55 bound the experimental errors. The PTW set obtained by calibration in sec. 3.3 (PTW Blumenthal RTI, red) gives excellent results.

\subsection{Surrogate model}

Figure 6 locates six representative material points of the simulation matching the experiment (i.e. $0.45 *$ Original). Their flow stress, plotted in the right hand side of figure 8(a) according strain, becomes the surrogate experiment. However, to avoid any intersection with the CT domain, only strains beyond 0.5 are considered here. The experimental standard deviation is arbitrarily set to $17 \%$ of the stress, an average value of the differences between the best $(0.45)$ and the upper- and lower-bounding coefficients (0.40 and 0.55). At the same time, pressures, temperatures, strains and strain rates from the six typical material points are used as inputs of a very fast analytical calculation of the PTW flow stress. Such a calculation conducted with any other specified PTW set becomes the surrogate simulation. In other words:

1. The surrogate experiment consists in six strainstress curves from six representative material points of the RTI simulated with $0.45 *$ Original (standard deviation of $17 \%$ of the stress);

2. The surrogate simulation is six strain-stress curves calculated with any PTW set thanks to the thermomechanical paths extracted from the six representative material points of the surrogate experiment.

This method strongly decreases the cost of an experimentsimulation comparison although it neglects the effects of flow stress variations on temperature, which are therefore expected to be of secondary importance.

\subsection{Priors and sensitivity analysis}

The priors of the next calibration are the same as the CT calibration's ones. First of all, we explore the sensitivity of the RTI to the seven parameters of table 1. The Morris's graph is displayed in figure 7 . The parameter $y_{0}$ has 


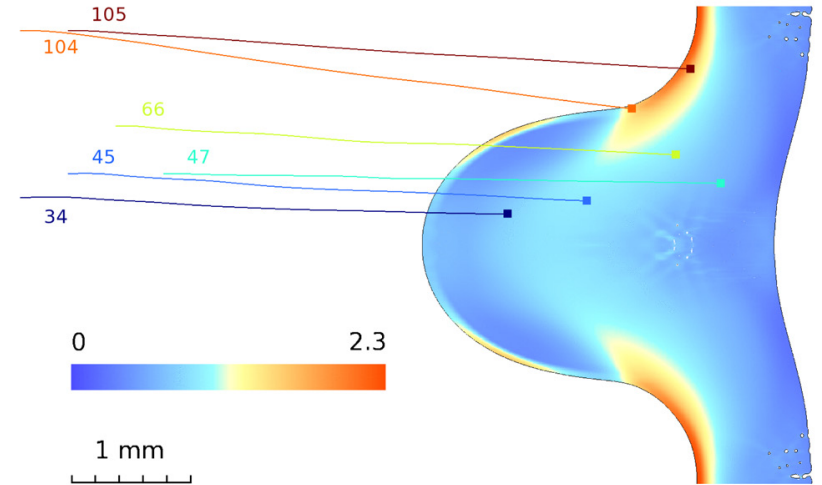

Fig. 6. Location and trajectories of six material points representative of the RTI. Their names (numbers) are used in figure 8 to identify the corresponding strain-stress curves. 2D-planar simulation performed with $0.45 *$ Original at $t=1.1 \mu \mathrm{s}$ ( $t_{0}$ being the HE's arrival time on Be's front face), i.e. $A / A_{0}=4.6$. Color scale: plastic strain.

a weak influence, which is consistent with the fact that it accounts for flow stress at low strain. This is also the case of $\theta$ and $\psi$, because they are linked to the strain hardening phenomenon playing a significant role only at low and moderate strain. The low sensitivity of $s_{\infty}$ indicates that the RTI growth occurs mainly out of the athermal plateau. Finally, although $\kappa$ and $y_{\infty}$ have moderate effects, they may be neglected regarding the importance of $s_{0}$. Thus, the Bayesian calibration should be performed only with $s_{0}$.

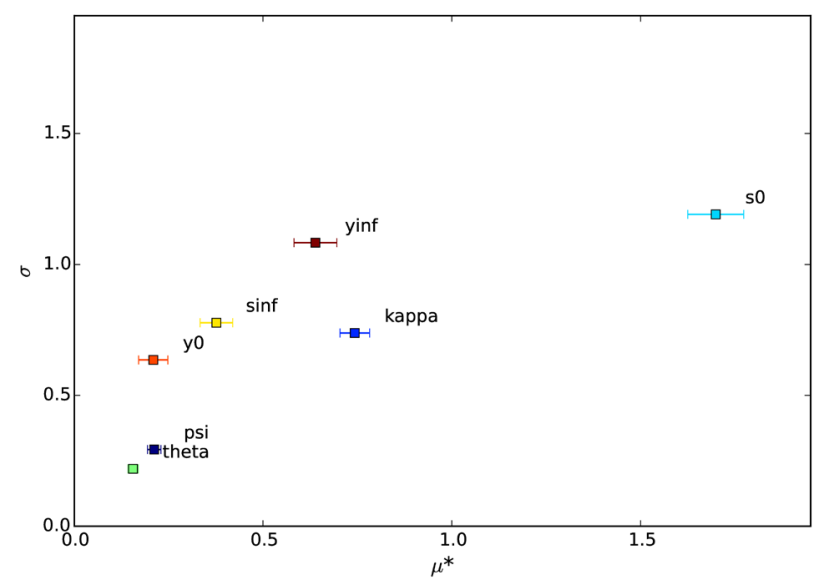

Fig. 7. Results of the Morris' sensitivity analysis for the surrogate RTI with priors from table $1 . \mu^{*}$ accounts for parameter's average sensitivity. $\sigma$ accounts for parameter's correlation to the others and non-linear sensitivity.

\subsection{Bayesian calibration and optimization}

A Bayesian calibration of $s_{0}$ is performed using the surrogate model described in sec. 3.1 and considering a Normal law for the experimental stress with a standard deviation of 17\% (see sec. 3.1). The six other parameters are fixed to their values optimized on the Blumenthal's compression tests database (see tables of figure $8(\mathrm{~b})$ ). As results, the right hand side of figure 8 (a) gives the posterior $95 \%$-CI of the strain-stress curves, which are very narrow and well bounded by the experimental ones.

The optimized value of $s_{0}$ is equal to 0.0041 . This new set of parameters, called PTW Blumenthal RTI, is then used in a hydrodynamic simulation of the Henry de Frahan's RTI whose growth factor according target's displacement is displayed in figure 5. Its good agreement with both experiment and PTW $0.45 *$ Original validates the use of a surrogate model in our Bayesian procedure.

\section{Discussion}

In the previous sections, we performed Bayesian calibrations and maximum likelihood processes for compression tests and Rayleigh-Taylor instabilities. For both, it provided optimized sets (tables of figure $8(\mathrm{~b})$ ) in very good agreement with experiments. Nonetheless, figure 8(c) evidences the inability of both PTW sets to match the experiment on which it has not been fitted. It also shows that no satisfying fit on the RTI (CT respectively) is possible in the posterior 95\%-CI from CT (RTI respectively). This point is rather well illustrated by figure $8(\mathrm{~d})$ where the two posterior probability densities of $s_{0}$ do not intersect. Therefore, it seems, in the specific case of beryllium, that a single fit of the PTW model cannot span RTI and CT loadings. The addition of an analytical model error to PTW would remove this restriction but would not clarify the potential reasons of this limitation which are the subject of the following discussion.

Although of heuristic nature, the PTW model best reproduces the behavior of metals deforming through thermally activated dislocations motion. The plasticity of beryllium is known to be much more complex than the BCC initially used to validate the PTW model, due to its hexagonal close-packed (HCP) crystal structure. More precisely, the main slip planes of beryllium (the basal $\{0002\}\langle 11 \overline{2} 0\rangle$ and prismatic $\{10 \overline{1} 0\}\langle 11 \overline{2} 0\rangle$ slip planes [13]) have very different activation stresses [14], and a possible strength anomaly [15]. The strength could thus be very different depending on the crystal orientation.

For non textured crystals, such peculiarities could be lumped in an averaged thermally activated behavior. For strongly deformed polycrystalline beryllium however, the texture could influence the behavior $[16,17]$ by changing the Schmid factor that governs the slip-slip competition, and thus the overall thermal sensitivity. For this reason, it may not be possible to fit a PTW model over such a large range of deformations, the activated slip systems being of very different nature for each texture.

Another possible deformation mechanism for beryllium is the onset and migration of twins [18] of the $\{10 \overline{1} 2\}\langle 10 \overline{1} 1\rangle$ system, a mechanism strongly dependent on the direction of loading with respect to the crystal orientation [19]. Since the twinning boundaries are strong barrier for dislocations (Hall-Petch type mechanism), the density of twins play a role is the hardening mechanisms, but with its own threshold and kinetics [20]. A model aiming at modeling plasticity and twinning should have dedicated parameters, which was not in the scope of the PTW model. 


\section{Conclusion}

This paper was an attempt to obtain a single PTW set of parameters bridging compression tests (CT) and RayleighTaylor instability (RTI) domains by means of a Bayesian approach. Using a surrogate model, sensivity analyzes and separated calibrations, we evidenced that such a purpose was unachievable for beryllium S200F. Finally, some physical explanation were discussed, all linked to microstructural strain mechanisms.

\section{Methods}

All the simulations presented in this paper, excepting hydrodynamic ones, have been performed with CALiXt, our OD plasticity code. It is based on the Python's standard scientific libraries, on pymc for Bayesian calibration and on SALib for sensitivity analysis.

The authors want to thank Laurianne Pillon and Grégory Robert for fruitful discussions and precious advice during the writing process.

\section{References}

1. J. F. Barnes et al., Journal of Applied Physics, 45, 727732 (1974)

2. H. S. Park et al., Physical Review Letters 104, 1-4 (2010)

3. H. S. Park et al., Physical Review Letters 114, 1-5 (2015)

4. M. T. Henry de Frahan et al., Journal of Applied Physics 117, 1-12 (2015)
5. B. L. Glushak et al., VANT Ser. Math. Model. Phys. Process. 2 (2012)

6. D. J. Steinberg et al., Journal of Applied Physics 51, 1498-1504 (1980)

7. D. J. Steinberg and C. M. Lund, Journal of Applied Physics 65, 1528-1533 (1989)

8. P. S. Follansbee and U. F. Kocks, Acta Metallurgica 36, 81-93 (1988)

9. D. L. Preston et al., Journal of Applied Physics 93, 211220 (2003)

10. W. R. Blumenthal et al., AIP Conference Proceedings, 411-414 (1998)

11. D. Montoya, $\mathrm{PhD}$ thesis of the University of Paris XIII (1991)

12. M. Fugate et al., Los Alamos National Laboratory Report LA-UR-05-3935 (2005)

13. A. Couret and D. Caillard, Philosophical Magazine, 59, 4, 783-800 (1988)

14. F. Aldinger, beryllium Science and Technology, 1 (1979)

15. A. Couret and D. Caillard, Philosophical Magazine, 59, 4, 801-819 (1988)

16. D.W. Brown et al, Int. Journal of Plasticity 29, 120135 (2012)

17. M. Knezevic et al, Int. Journal of Plasticity 49, 185198 (2013)

18. F. Mompiou et al., Journal of Material Research 32, 24, 4616-4625 (2017)

19. D.W. Brown et al, Metall. Trans. A 36A, 929-939 (2005)

20. M. Messner et al, Modelling Simul. Mater. Sci Eng. 25, 044001 (2017) 


\section{Compression tests (CT)}

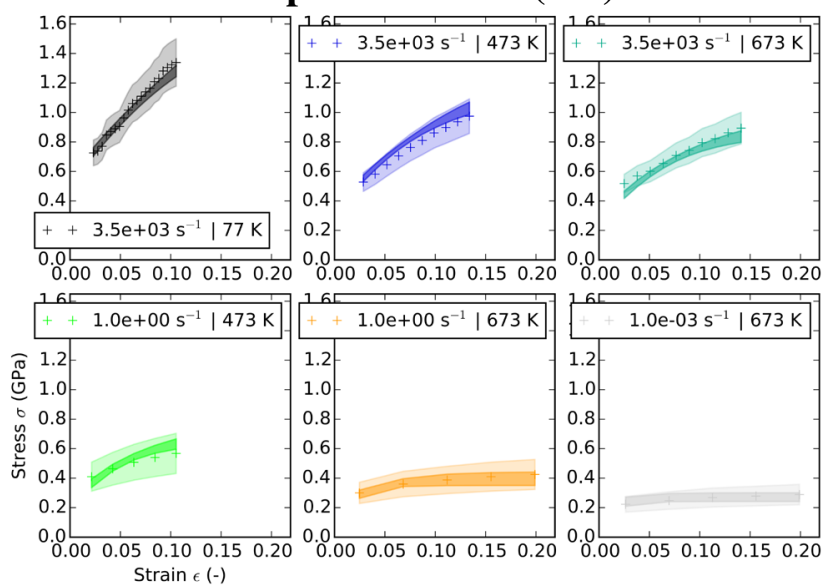

Rayleigh-Taylor instability (RTI)

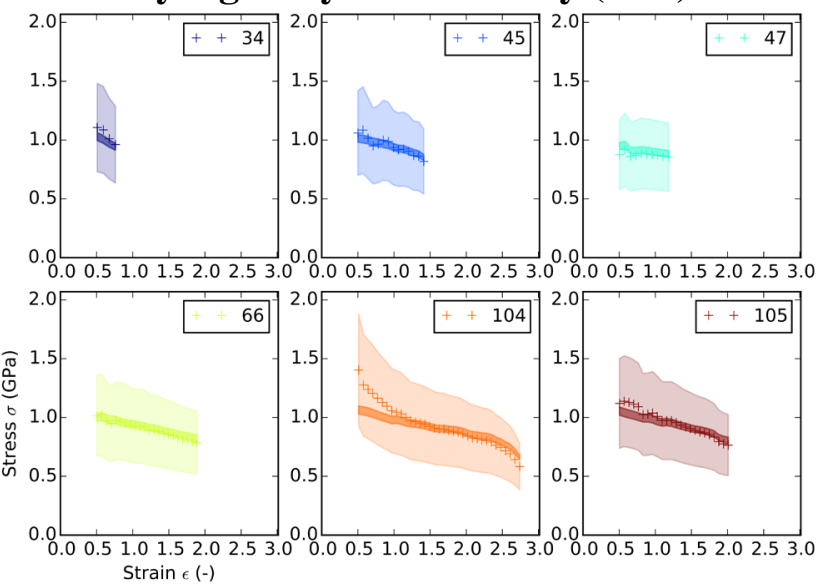

(a) Comparison between experimental (light shadows) and calibrated (heavy shadows) 95\%-confidence intervals. (+): experiment.

PTW Blumenthal CT

\begin{tabular}{ccccccc}
\hline \multicolumn{8}{c}{$s_{0}$} & $s_{\infty}$ & $\kappa$ & $y_{0}$ & $y_{\infty}$ \\
\hline- & - & 0.0070 & 0.0007 & 0.17 & 0.0017 & 0.0002 \\
- & - & 0.0110 & 0.0010 & 0.23 & 0.0020 & 0.0006 \\
\hline 0.03 & 0 & 0.0800 & 0.0008 & 0.19 & 0.0019 & 0.0004 \\
\hline
\end{tabular}

PTW Blumenthal RTI

\begin{tabular}{ccccccc}
\hline$\theta$ & $\psi$ & $s_{0}$ & $s_{\infty}$ & $\kappa$ & $y_{0}$ & $y_{\infty}$ \\
\hline- & - & 0.0039 & - & - & - & - \\
- & - & 0.0042 & - & - & - & - \\
\hline 0.03 & 0 & 0.0041 & 0.0008 & 0.19 & 0.0019 & 0.0004 \\
\hline
\end{tabular}

(b) From top to bottom: 95\%-confidence intervals and maximum likelihoods of the calibrated parameters. Italic text: values reused from CT.

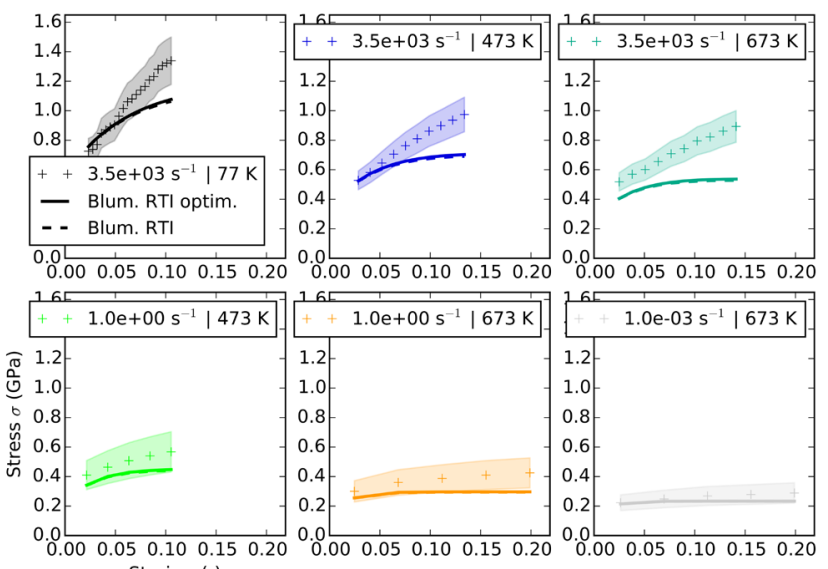
Strain $\epsilon(-)$
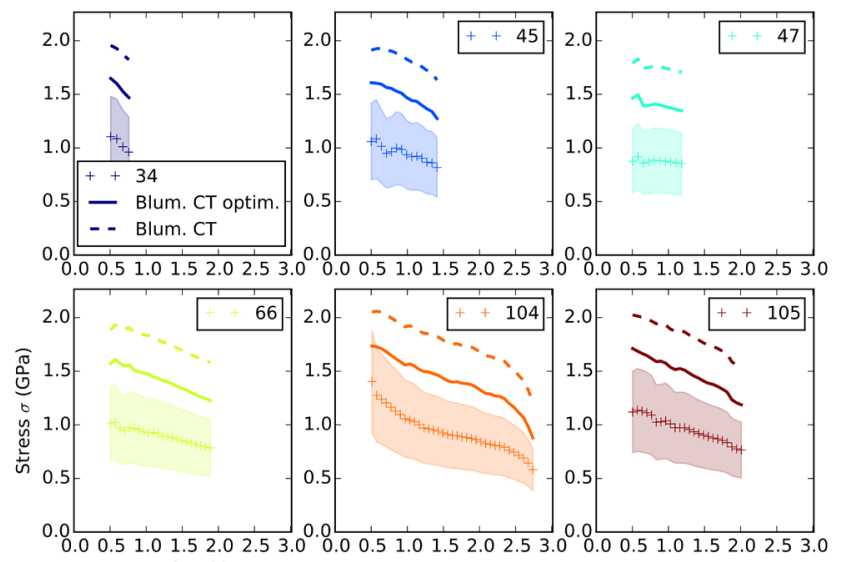
Strain $\epsilon(-)$

(c) $(+)$ : experiment. Shadows: experimental 95\%-confidence intervals. (--): simulation performed with the posterior set of the other domain (CT or RTI). (-): best fit in the posterior 95\%-CI of the other domain (CT or RTI).

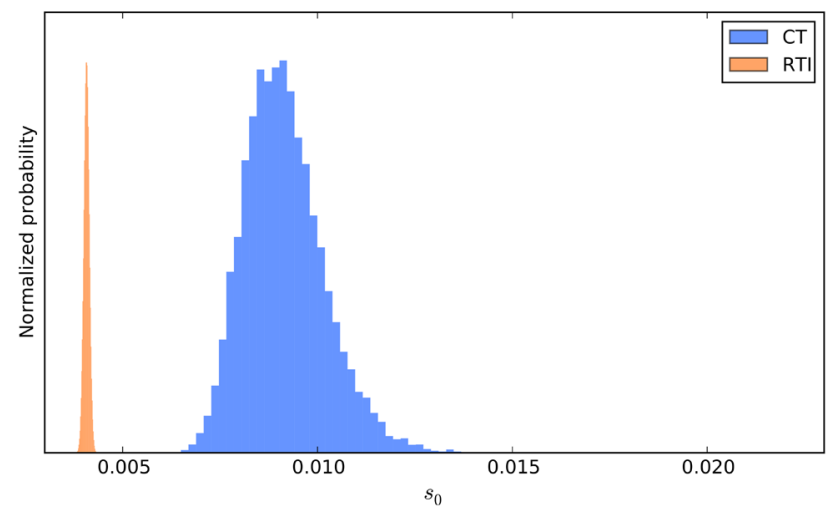

(d) Posterior probability densities of $s_{0}$ for compression tests (right, blue) and Rayleigh-Taylor instability (left, orange).

Fig. 8. Summary of the Bayesian crossed-data analysis on compression tests and Rayleigh-Taylor instability. Left column: results for compression tests (CT). Right column: Rayleigh-Taylor instability (RTI). 